\title{
Determinação do ciclo estral em catetos Pecari tajacu: aspectos colpocitológicos e clínicos
}

\author{
Diva Anelie de Araujo GUIMARÁES ${ }^{1}$, Sylvia Cristina Garcia de GARCIA², Yvonnick LE PENDU³, Natália \\ Inagaki de ALBUQUERQUE ${ }^{4}$
}

\begin{abstract}
RESUMO
Este trabalho teve por objetivo estudar o ciclo estral do cateto, aplicando a colpocitologia como método diagnóstico. As amostras da citologia vaginal foram coletadas em dez fêmeas adultas, durante três vezes na semana, por seis meses. Verificou-se que a duraçáo média do ciclo estral para esta espécie foi de 28,45 $\pm 5,45$ dias. Observou-se diferença estatística $(\mathrm{p}<0,01) \mathrm{em}$ relaçáo à frequência dos diferentes tipos celulares na mesma fase do ciclo estral. No proestro foi verificado o aumento de células intermediárias e superficiais. A fase de estro caracterizou-se pela elevação significativa de células superficiais em relação aos demais tipos celulares e pela ausência de leucócitos. Durante este período, a genitália externa estava hiperêmica, edemaciada e com muco. No metaestro houve um decréscimo de células superficiais, quando comparado com o proestro e com o estro, e uma elevação significativa de células intermediárias, presença de leucócitos, de células de metaestro e de foam. Na fase de diestro, houve um aumento de células intermediárias, e um decréscimo no número de leucócitos. Conclui-se que, por meio da colpocitologia, é possível diferenciar as fases do ciclo estral em catetos.
\end{abstract}

PALAVRAS-CHAVE: citologia vaginal, reprodução, Pecari tajacu, Tayassuidae, Amazônia

\section{Determination of the estrous cycle in collared peccary Pecari tajacu: colpocytological and clinical aspects}

\section{ABSTRACT}

The main objective of this work was to study the estrous cycle of collared peccary by vaginal cytology. Samples were collected in 10 adult females, three times a week, for six months. The estrous cycle period for this specie was $28.45 \pm 5.45$ days. We observed a significant difference $(\mathrm{p}<0.01)$ between the cell types in the same phase of the oestrous cycle. During the proestrus phase, the exfoliative cytology arrangement showed high frequency of intermediate and superficial cells. The estrus phase had elevated rates of superficial cells, when compared to the other types, and an absence of leukocytes. During this phase, the external genitalia were reddish, tumescent; and the cervical mucus was evident. In the metaestrus phase, we observed a decrease of superficial cells, as well as the highest indices of intermediate cells, an increase of leukocytes and the presence of metestrus and foam cells. In the diestrus phase, the intermediate cells increased and leukocytes decreased. In conclusion, the present study suggests that it is possible to differentiate the phase of estrous cycle by vaginal cytology.

KEYWORDS: vaginal cytology, reproduction, Pecari tajacu, Tayassuidae, Amazonia. 


\section{INTRODUÇÃO}

A citologia esfoliativa estuda as características das células que descamam de diversos órgãos do corpo (Junqueira e Carneiro 2000). Quando essa técnica é aplicada às células do epitélio vaginal, denomina-se de colpocitologia.

O epitélio vaginal apresenta mudanças bem definidas durante o ciclo estral, as quais ocorrem devido ao equilíbrio dos hormônios sexuais (estrógeno e progesterona) no organismo (Hafez 2004). Os tipos celulares observados neste epitélio indicam diferentes estágios de diferenciação celular. Carvalho (1993) dividiu este epitélio nas seguintes camadas e tipos celulares: 1- Basal profunda (célula basal); 2- Basal superficial (célula parabasal); 3- Intermediária (célula intermediária); 4- Superficial (célula superficial nucleada ou anucleada).

A colpocitologia é utilizada no estudo reprodutivo de animais domésticos como: cães (Roszel 1975), bovinos (Miroud e Noakez 1990), suínos (Carabin et al. 1995) e ovinos (Porto et al. 2007). Esta técnica também é aplicada em animais silvestres, por exemplo, em cutia (Dasyprocta prymnolopha), ratão do banhado (Myocastor coypus), capivara (Hydrochoerus hydrochaeris) e paca (Cuniculus paca) (Guimarães et al. 1997; Felipe et al. 2001; Nogueira et al. 2005; Barbosa et al. 2007; Guimarães et al. 2008). Pode servir para a observação das fases do ciclo estral (proestro, estro, metaestro, diestro), para o conhecimento da data aproximada da parição por confirmaçáo de cópula, para o diagnóstico auxiliar de problemas de infertilidade e para as determinaçóes de enfermidades como tumores vaginais, vaginites e piometra (Feldman e Nelson 1987).

$\mathrm{O}$ uso da colpocitologia no manejo reprodutivo de animais silvestres de interesse comercial como o cateto (Pecari tajacu) (Figura 1) é útil para o controle da fase fértil, entre outras aplicações, possibilitando a utilização de biotécnicas, como a inseminação artificial, contribuindo de maneira positiva aos programas de reprodução.

O cateto é uma das espécies silvestres mais consumida na região rural do norte do Brasil (Bonaudo et al. 2005) e a sua criação comercial é prevista na legislação do Instituto Brasileiro do Meio Ambiente e dos Recursos Naturais Renováveis (IBAMA). O estudo sobre a reprodução destes animais é desenvolvido pela Universidade Federal do Pará em convênio com a Embrapa Amazônia Oriental (Silva et al. 2002b; Guimarães et al. 2005; Mayor et al. 2006; Mayor et al. 2007a; Mayor et al. 2007b; Kahwage et al. 2008; Costa et al. 2010) e visa o aumento da produção em cativeiro, contribuindo para a segurança alimentar, a conservação da espécie e a geração de emprego e renda nesta região. Todavia, são necessários estudos que visem o manejo reprodutivo correto desta espécie, especialmente na época fértil.

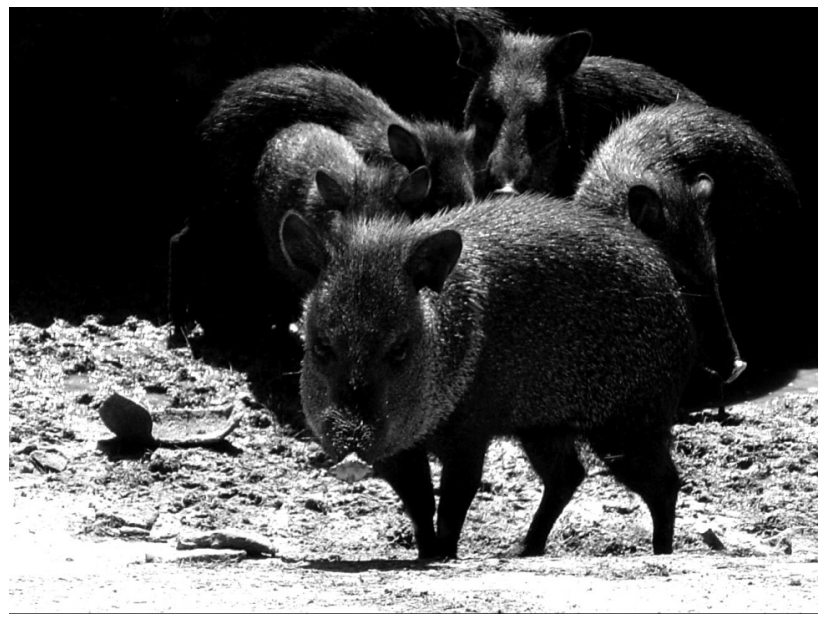

Figura 1 - Cateto (P. tajacu) do criatório de animais silvestres da Embrapa Amazônia Oriental.

Sobre o ciclo estral do cateto, Barbella (1993), Mauget et al. (1997) e Sowls (1997) observaram períodos de 22,38 \pm 1,73 dias (17 a 25 dias), 27,8 \pm 1,5 dias (23 a 34 dias) e 22 a 24 dias, respectivamente. Com relação às análises colpocitológicas realizadas em catetos, Barbella (1993) observou no estro o aumento de células superficiais, no metaestro a presença de leucócitos e hemácias e, em seguida, o aparecimento de células parabasais. Contudo, Silva et al. (2002a) afirmaram que a célula intermediária predomina em todo o ciclo, o que dificulta o diagnóstico das fases, não sendo possível a sua determinação através da observação das frequências celulares. Por outro lado, Mayor et al. (2007a) observaram a predominância de células superficiais e intermediárias durante quatro dias no estro, as quais foram reduzindo em seguida. Como não há um consenso entre os autores, é fundamental o estabelecimento do período do ciclo estral em catetos, assim como do perfil colpocitológico das diferentes fases.

$\mathrm{Na}$ Amazônia brasileira, o uso de um método diagnóstico rápido, eficiente e de menor custo como a colpocitologia é interessante para se ter o controle reprodutivo da fêmea de cateto, uma espécie poliéstrica contínua (Mayor et al. 2007b), com maturidade sexual alcançada aos sete meses (NogueiraFilho e Nogueira 2004). Nesse contexto, este trabalho teve por objetivo determinar o período do ciclo estral de cateto por meio das observaçóes colpocitológicas e dos aspectos clínicos da genitália externa em cada fase do ciclo, permitindo conhecer a ação endócrina no epitélio vaginal durante todo este período.

\section{MATERIAL E MÉTODOS}

Os catetos nascidos no criatório científico da Embrapa Amazônia Oriental, situado na Unidade de Pesquisa Animal Senador Álvaro Adolpho, em Belém-Pará, Brasil (1²8'S e $48^{\circ} 27^{\prime} \mathrm{O}$ ), foram mantidos em baias de $21 \mathrm{~m}^{2}$ e $36 \mathrm{~m}^{2}$, em 
grupos familiares de quatro a nove indivíduos, entre adultos e filhotes.

Este estudo foi realizado em 10 fêmeas adultas nulíparas, com idade variando entre 10 e 33 meses, durante seis meses (março a agosto). As amostras foram colhidas três vezes na semana, sendo processadas e analisadas no Laboratório de Reprodução Animal, Instituto de Ciências Biológicas, Universidade Federal do Pará. Para a colheita foram utilizados suabes estéreis, introduzidos até a parte medial do orifício vaginal, seguindo o método utilizado em cutias e pacas (Guimarães et al. 1997; Guimarães et al. 2008).

O esfregaço foi fixado em álcool $95 \%$ e colorado pelo método clássico de Papanicolau (NEWPROV, Paraná, Brasil). As lâminas foram observadas ao microscópio óptico em objetivas de 40x, para a contagem de 100 células, que foram classificadas, de acordo com Carvalho (1993) em basais, parabasais, intermediárias e superficiais. Segundo este autor, as células basais são esféricas e possuem núcleo central ocupando cerca de $2 / 3$ do citoplasma; as parabasais são esféricas ou ovaladas, possuem o núcleo esférico e central, o qual corresponde a $1 / 2$ da área celular; as intermediárias são arredondadas ou poligonais, com núcleo ovalado ou esférico, preenchendo $1 / 4$ a $1 / 5$ da área celular; as superficiais são poligonais, com núcleo pequeno e central. Sendo que a proporção dos tipos celulares serviu para determinar a fase do ciclo estral, da mesma forma como foi realizado em cutias e pacas (Guimaráes et al. 1997; Guimarães et al. 2008).

Foram verificados também os aspectos clínicos da genitália externa. $\mathrm{O}$ exame da vulva (comissuras e lábios) foi realizado antes da colheita, sendo observada a presença ou náo de edema e hiperemia neste local. No vestíbulo vaginal foi observada a presença ou não de muco cervical. Estes parâmetros também serviram para identificaçáo das diferentes fases do ciclo estral, seguindo os critérios adotados por Mayor et al. 2007a.

Para a análise dos dados foram realizados testes estatísticos descritivos (média e desvio padrão), e para a frequência das células em cada fase do ciclo estral foi empregada a análise de variância (ANOVA) e pós-teste de Tukey com nível de significância de 5\%. Todas as análises foram feitas através do software BioEstat 3.0 (Ayres et al. 2003).

\section{RESULTADOS E DISCUSSÃO}

Foram analisados 54 ciclos com duração média de 28,45 \pm 5,45 dias e amplitude de variação entre 18 e 40 dias. As diferenças e as similaridades entre os resultados encontrados na literatura e os do presente trabalho podem estar relacionadas a fatores ambientais, como a temperatura e a luminosidade, já que Barbella (1993) e Sowls (1997) realizaram os estudos em locais geograficamente mais distantes do presente trabalho (Maracay-Venezuela e Texas-EUA) e Mauget et al. (1997) em uma região bem mais próxima (Guiana Francesa). Além disso, fatores distintos, como a vida reprodutiva, a faixa etária, o fato de a fêmea ser mantida isolada ou em grupo familiar, a quantidade de indivíduos por recinto e o tipo de manejo utilizado, podem influenciar na duraçáo deste período. Neste caso, as relaçóes sociais entre os animais, as quais causam ou não estresse, e os fatores químicos (ferormônios) podem agir em nível endócrino, ampliando ou encurtando o ciclo estral.

As análises colpocitológicas mostraram a presença de células superficiais, intermediárias, parabasais e basais visualizadas em todas as fases do ciclo estral (Tabela 1).

Com relação à morfologia, as células superficiais eram poligonais, com o citoplasma acidófilo, sendo anucleadas ou nucleadas (com o núcleo intacto ou picnótico). As células intermediárias possuíam formato ovalado ou esférico, citoplasma basófilo, abundante e núcleo central. As células parabasais eram ovaladas ou esféricas, com citoplasma basófilo, reduzido e núcleo central ou periférico. As basais eram esféricas, com núcleo hipercromático ocupando quase a totalidade celular.

Essas observaçóes são semelhantes às descrições de Oba (1979) para suínos e de Silva et al. (2002a) para catetos. Contudo, a primeira autora classificou as células intermediárias em pequena, média e grande, e os segundos autores não citaram as células basais.

Com relação à frequência celular, apesar de não ter sido observada diferença estatística $(p>0,05)$ entre as fases do ciclo estral, verificou-se $(\mathrm{p}<0,01)$ dentro de cada fase (Tabela 1$)$.

$\mathrm{Na}$ fase de proestro, inicialmente as células intermediárias estavam em maior quantidade; posteriormente houve um aumento de superficiais nucleadas. A média das superficiais e intermediárias foi estatisticamente significativa $(\mathrm{p}<0,01)$ com relação às parabasais e basais. Não foi observada a presença de leucócitos. Esta fase teve duração média de 6,1 \pm 0,7 dias, com amplitude de variação de seis a nove dias.

$\mathrm{O}$ estro caracterizou-se pelo aumento significativo $(\mathrm{p}<0,01)$ na quantidade de células superficiais (anucleadas e nucleadas), em relação aos demais tipos celulares, e pela ausência de leucócitos (Figura 2). Esta fase teve duração de

Tabela 1 - Distribuição dos tipos celulares durante as fases do ciclo estral no cateto.

\begin{tabular}{lcccc}
\hline Fase & $\begin{array}{c}\text { Superficiais } \\
(\%)\end{array}$ & $\begin{array}{c}\text { Intermediárias } \\
(\%)\end{array}$ & $\begin{array}{c}\text { Parabasais } \\
(\%)\end{array}$ & $\begin{array}{c}\text { Basais } \\
(\%)\end{array}$ \\
\hline Proestro & $50,3 \pm 17,8^{\mathrm{a}}$ & $43,8 \pm 13,5^{\mathrm{a}}$ & $4,8 \pm 6,1^{\mathrm{b}}$ & $1,1 \pm 2,2^{\mathrm{b}}$ \\
Estro & $66,6 \pm 13,7^{\mathrm{a}}$ & $30,7 \pm 10,9^{\mathrm{b}}$ & $1,94 \pm 3,0^{\mathrm{c}}$ & $0,7 \pm 0,9^{\mathrm{c}}$ \\
Metaestro & $40,3 \pm 15,2^{\mathrm{a}}$ & $55,7 \pm 11,8^{\mathrm{b}}$ & $3,3 \pm 3,0^{\mathrm{c}}$ & $0,7 \pm 1,0^{\mathrm{c}}$ \\
Diestro & $43,6 \pm 14,7^{\mathrm{a}}$ & $49,7 \pm 12,1^{\mathrm{b}}$ & $5,1 \pm 4,9^{\mathrm{c}}$ & $1,6 \pm 2,4^{\mathrm{c}}$ \\
\hline
\end{tabular}

Os valores estão expressos em médias.

Letras diferentes na mesma linha indicam diferença significativa $(p<0,01)$. ANOVA. 
3,0 \pm 0,4 dias, variando de dois a quatro dias, aproximandose dos períodos observados por Barbella (1993) (3 a 5 dias), Mauget et al. (1997) ( $4 \pm 2,9$ dias), Sowls (1997) (3,5 a 4,8 dias) e Mayor et al. (2007a) (4,4 $\pm 2,6$ dias). Todavia, são necessários estudos etológicos para definir com mais precisão o período de aceitação ao macho, nesta espécie.

O metaestro caracterizou-se por um decréscimo de células superficiais, quando comparado com o proestro e com o estro, por uma elevação significativa $(\mathrm{p}<0,01)$ de células intermediárias e pela presença de leucócitos, de células do metaestro e de foam. Barbella (1993) afirmou que nesta fase havia a presença de eritrócitos, os quais, porém, não foram observados neste trabalho. As células intermediárias foram estatisticamente significantes $(\mathrm{p}<0,01)$ quando comparadas aos outros tipos celulares. Esta fase teve duração média de $2,0 \pm 0,4$ dias, variando de três a quatro dias.

O diestro caracterizou-se pelo predomínio de células intermediárias, mostrando diferença estatística $(\mathrm{p}<0,01)$ entre os demais tipos celulares. Foi observado um número expressivo de degeneração celular, além de um decréscimo no número de leucócitos (Figura 3). A duração em média foi de 10,1 $\pm 2,1$ dias, com variação de cinco a onze dias.

A característica diferencial do proestro para o estro foi o aumento de células superficiais. Desta última fase para o metaestro foi a elevação de intermediárias, a presença de leucócitos, de células do metaestro e de foam. Do metaestro para o diestro foi observada a diminuição de leucócitos e a elevação de degeneração celular. Desta última fase para o proestro, houve, novamente, o aumento de células superficiais, o que demonstra a ação estrogênica no epitélio vaginal. Assim, estas observaçóes podem ser utilizadas como indicativo para o diagnóstico destas fases.

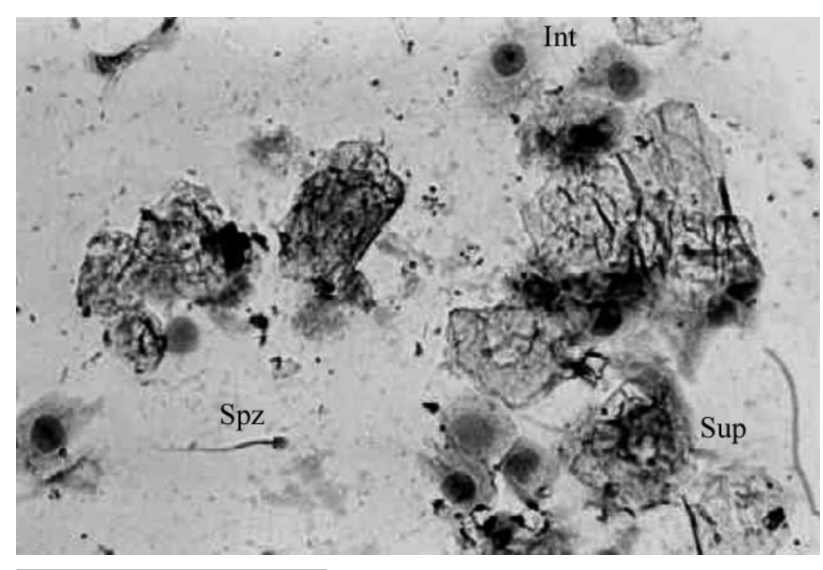

Figura 2 - Citologia esfoliativa durante a fase estrogênica. Sup: Célula superficial; Int: Célula Intermediária; Spz: Espermatozóide - 40 x.

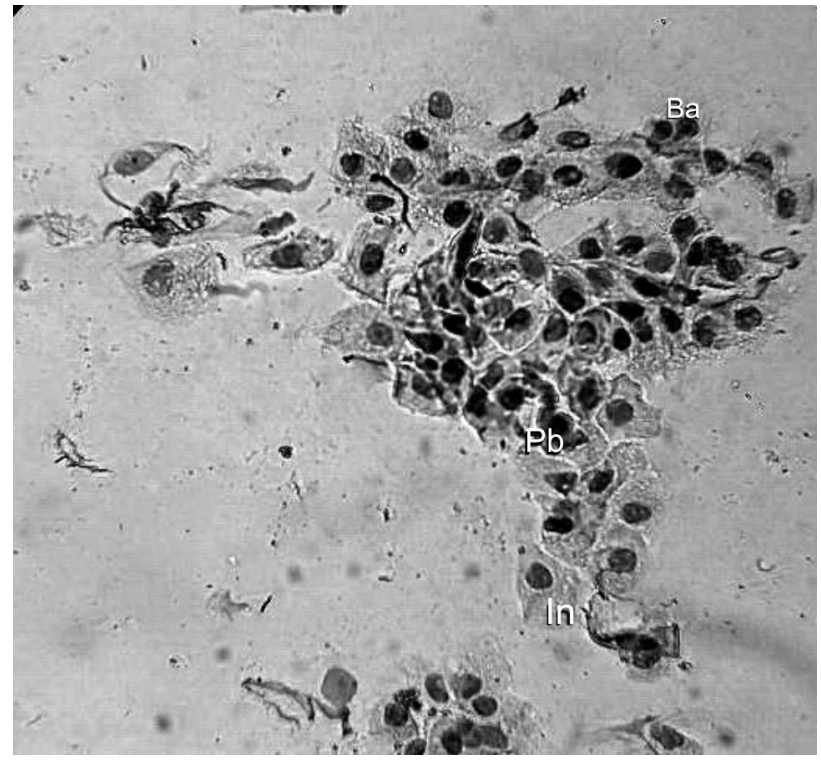

Figura 3 - Citologia esfoliativa durante a fase progesterônica. In: Célula intermediária; $\mathrm{Pb}$ : Célula parabasal; Ba: Célula basal - 40x.

Com relação aos sinais clínicos da genitália externa, foi observado que no período próximo ao estro houve hiperemia e edema, sendo que nas outras fases apresentou-se pálida e sem edema. A presença de muco cervical fluido e transparente só foi notada na fase de estro, mas em pouca quantidade. Esses resultados corroboram as observaçóes de Mayor et al. (2007a).

\section{CONCLUSÕES}

Os resultados do presente trabalho demonstram que as células superficiais e intermediárias estão presentes em número maior em todo o ciclo estral, mas na fase de estro as superficiais são significativamente mais elevadas, assim como no metaestro e diestro são as intermediárias. Portanto, para o correto diagnóstico das fases, é necessário considerar os outros aspectos, como a presença ou ausência de leucócitos, a relação entre o número de células intermediárias e superficiais, além dos sinais clínicos da genitália externa.

Pode-se afirmar que a colpocitologia é uma técnica eficiente para a caracterizaçáo do ciclo estral em catetos, permitindo, com segurança satisfatória, a detecção das diferentes fases nessa espécie. Dessa forma, usá-la como rotina no manejo destes animais será um auxílio seguro nos programas de reprodução.

\section{AGRADECIMENTOS}

Ao Conselho Nacional de Desenvolvimento Científico e Tecnológico (TAYATAJA-CNPq n471705/03) e à Secretaria Executiva de Ciência, Tecnologia e Meio Ambiente 
(SECTAM/FUNTEC/UFPA n ${ }^{\circ} 307412$ ) pelo apoio financeiro concedido ao projeto.

\section{BIBLIOGRAFIA CITADA}

Ayres, M.; Ayres Jr, M.; Ayres, D.L.; Santos, A.S. 2003. Bioestat 3.0: Statistical applications in the areas of biological and medical sciences. Sociedade Civil Mamirauá/MCT/CNPq, Belém, Pará, Brazil. 290 pp. (in Portuguese).

Barbella, S.L. 1993. Determination of the estrous cycle in the collared peccary (Tayassu tajacu). Revista da Facultad de Agronomia (Maracay), 9: 167-174 (in Spanish, with abstract in English).

Bonaudo, T.; Le Pendu, Y.; Faure, J.F.; Quanz, D. 2005. The effects of deforestation on wildlife along the transamazon highway. European Journal of Wildlife Research, 5: 199-206.

Barbosa, L.P.; Rodrigues, M.V.; Neves, M.M.; Morais, D.B.; Melo, B.E.S.; Balarini, M.K.; Coelho, C.D.P.; Mendonça, C. 2007. Characterization of colpocytology in capybaras (Hydrochoerus hydrochaeris). Revista Brasileira de Saúde e Produção Animal, 8: 258-266 (in Portuguese, with abstract in English).

Carabin H.; Desnoyers, M.; Vaillancourt, D.; Martineau, G.P. 1995. Influence of vulvar hygiene on cytology of vaginal smears after sham artificial insemination in sows. Canadian Journal of Veterinary Research, 59: 193-196.

Carvalho, G. 1993. Study of vaginal and cervical epithelia and their exfoliated cells. Relations and interpretations, p. 23-28. In: Carvalho, G. (Ed). Cytology of the Female Genital Tract. Atheneu, São Paulo, Brazil (in Portuguese).

Costa, G.M.J.; Leal, M.C.; Silva, J.V.; Cassia, A.; Ferreira, S.; Guimarães, D.A.; Franca, L.R. 2010. Spermatogenic cycle length and sperm production in a feral pig species (collared peccary, Tayassu tajacu). Journal of Andrology, 31: 221-230.

Feldman, E.C.; Nelson, R.W. 1987. Canine female reproduction, p. 399-418. In: Feldman, E.C.; Nelson R.W. (Eds). Canine and Feline Endocrinology and Reproduction. Saunders Company, Philadelphia, USA.

Felipe, A.E.; Carbodevila, J.; Callejas, S. 2001. Characterization of estrous cycle of the Myocastor coypus (coypu) by means of exfoliative colpocytology. Journal of Neotropical Mammalogy, 8: $129-137$

Guimarães, D.A.; Albuquerque, N.I.; Le Pendu, Y.; Silva, J.V.D.; Dias, H.L.T. 2005. Productive and reproductive management of the collared peccary (Tayassu tajacu) in captivity. Revista de Ciências Agrárias, 43: 1-5 (in Portuguese).

Guimarães, D.A.; Bastos L.V.; Ferreira, A.C.S.; Luz-Ramos R.S.; Ohashi, O.M.; Ribeiro, H.L. 2008. Reproductive characteristics of female paca (Agouti paca) bred in captivity. Acta Amazonica, 38: 531-538 (in Portuguese, with abstract in English).

Guimarães, D.A.; Moreira, D.; Vale, W.G. 1997. Determination of the reproductive cycle (Dasyprocta prymnolopha) through the smear diagnosis. Acta Amazonica, 27: 55-64 (in Portuguese, with abstract in English).
Hafez, E.S.E. 2004. Animal Reproduction. Manole, São Paulo, Brazil. 582 pp (in Portuguese).

Junqueira, L.C.; Carneiro, J. 2000. Female reproduction system, p. 382-384. In: Junqueira, L.C.; Carneiro, J. (Eds). Basic Histology. Guanabara e Koogan, Rio de Janeiro, Brazil (in Portuguese).

Kahwage, P.R.; Garcia, A.R.; Bartha, M.M.P.; Guimarães, D. A.; Luz, R.S.; Dias, H.L.T.; Albuquerque, N.I.; Ohashi, O. 2008. Development of protocol for sedation and analgesia in peccary (Tayassu tajacu) for collection of semen, p. 1-6. In: 35 Brazilian Congress of Veterinary Medicine, Gramado, RS, Brazil (in Portuguese).

Mauget, R.; Feer, F.; Henry, O.; Dubost, G. 1997. Hormonal and behavioural monitoring of ovarian cycles in peccaries. Zeitschrift fur Saugetierkunde, 62: 145-149.

Mayor, P.; Gálvez, H.; Guimarães, D.A.; Lópes-Gatius, F.; LópezBéjar, M. 2007a. Serum estradiol-17, vaginal cytology and vulval appearance as predictor of estrus cyclicity in the female collared pecary (Tayassu tajacu) from the eastern Amazon region. Animal Reproduction Science, 97: 165-174.

Mayor, P.; Guimarães, D.A.; Le Pendu, Y.; Silva, J.V.; Jori, F.; López-Béjar, M. 2007b. Reproductive performance of captive collared peccaries (Tayassu tajacu) in the eastern Amazon. Animal Reproduction Science, 102: 87-97.

Mayor, P.; Guimarães, D.A.; López-Gatius, F.; López-Béjar, M. 2006. First postpartum estrus and pregnancy in the female collared peccary (Tayassu tajacu) from the Amazon. Theriogenology, 66: 2001-2007.

Miroud, K.; Noakes, D.E. 1990. Exfoliative vaginal cytology during the oestrous cycle of the cow, after ovariectomy, and after exogenous progesterone and oestradiol-17 $\beta$. British Veterinary Journal, 146: 387-397.

Nogueira-Filho, S.L.G.; Nogueira, S.S.C. 2004. Captive breeding programs as an alternative for wildlife conservation in Brazil, p. 171-190. In: Silvius, K.M.; Bodmer, R.; Fragoso, J.M.V. (Eds.) People and nature: wildlife conservation in South and Central America. Columbia University Press, New York, USA.

Nogueira, T.M.R.; Toniollo, G.H.; Giannoni, M.L. 2005. Estrous cycle colpocytology in captive pacas (Agouti paca, Linnaeus, 1766). Ars Veterinaria, 21: 209-214

Oba, E. 1979. Histological and cytological vaginal mucosa of sows during the phases of the estrous cycle. Dissertação de Mestrado, Universidade Federal de Minas Gerais, Belo Horizonte. 58 pp (in Portuguese). [Unpublished]

Porto, R.R.; Cavalcante, T.V.; Dias, F.E.; Rocha, J.M.; Souza, J.A. 2007. Vaginal cytological profile of Santa Inês ewes to monitor estrous cycle. Ciência Animal Brasileira, 8: 521-527 (in Portuguese, with abstract in English)

Roszel, J.F. 1975. Genital cytology of the bitch. Veterinary Scope, 19:3-15.

Silva, J.M.; Lima, K.F.; Nunes, J.F.; Soares, J.B. 2002a. Characterization of the vaginal epithelium in collared peccary 
ACTA

AMAZONICA

Determinação do ciclo estral em catetos Pecari tajacu:

aspectos colpocitológicos e clínicos

raised in captivity. Revista Brasileira de Reprodução Animal, 5: 174-176 (in Portuguese).

Silva, J.V.; Cardoso, D.; Guimarães, D.A.; Albuquerque, N.; Le Pendu, Y.; Ohashi, O. 2002b. Reproductive biology of female collared peccary (Tayassu tajacu) raised in captivity in the Amazon. Revista Brasileira de Reprodução Animal, 5: 180-182 (in Portuguese).
Sowls, L.K. 1997. Javelinas and other peccaries. Their biology, management and use. Texas A\&M University Press, Texas, USA. $325 \mathrm{pp}$.

Recebido em 23/08/2010

Aceito em 17/12/2010 